\title{
Gravitational waves from precessing engines in GRBs
}

\section{Gustavo E. Romero}

Instituto Argentino de Radioastronomía (IAR), CCT La Plata (CONICET), Argentina, and Facultad de Ciencias Astronómicas y Geofísicas, Universidad Nacional de La Plata(UNLP), Paseo del Bosque s/n, 1900, La Plata, Argentina

E-mail: romero@fcaglp.unlp.edu.ar

\section{Matías M. Reynoso}

Instituto de Investigaciones Físicas de Mar del Plata (CONICET - UNMdP), Universidad Nacional de Mar del Plata, Dean Funes 3350, (7600) Mar del Plata, Argentina

E-mail: mreynoso@mdp.edu.ar

\section{Hugo R. Christiansen}

State University of Ceará, Physics Dept., Av. Paranjana 1700, 60740-000 Fortaleza - CE, Brazil; Universidade Estadual Vale do Acaraú, Av. da Universidade 850, 62040-370 Sobral - CE, Brazil

E-mail: hugochristiansen@yahoo.com.br

In the framework of a transient accretion disk at the core of a gamma-ray burst we compute possible periods of Lense-Thirring precession. Next, we evaluate the putative gravitational waves associated with such dynamical setup. Assuming a characteristic time-profile for the gamma-ray emission of a disk-jet system, we obtain light-curves presenting a time microstructure similar to that reported in some GRB events. After adjustment of the parameters out of two specific GRBs we evaluate the detectability of the gravitational waves produced by the precession of this accretion disk. As a conclusion, our analysis shows that some GRBs are likely to be probed with Advanced LIGO.

25th Texas Symposium on Relativistic Astrophysics - TEXAS 2010

December 06-10, 2010

Heidelberg, Germany 


\section{Introduction}

It is widely thought that gamma-ray bursts (GRBs) are stellar events produced out of a central object that consists of a massive black hole with a transient, hot and dense accretion disk. Such an engine might result from a failed supernova or collapsar (e.g. [1]) or the merging of two compact objects (e.g. [2]). Apparently, the accretion of matter onto the black hole is the energy source of the system. Part of this energy is ejected through an ephemeral relativistic bipolar jet, normal to the midplane of the disk, radiating an extremely intense burst of gamma-rays. The duration of these gamma-ray pulses ranges from milliseconds up to minutes. This picture is expected to underly most of the observed GRB light-curves.

The usual explanation for the temporal structure of such light curves relays on the formation of shock waves that convert bulk kinetic energy into relativistic energy of the particles in the jets. Charged particles then cool by synchrotron and inverse Compton emission. The shocks can be either internal to the jet and produced by colliding shells with different Lorentz factors (e.g. [3, 4, 5]) or the result of interactions with the external medium (e.g. [6]).

Nevertheless, some peculiar GRB light curves are difficult to explain by means of shocks only, particularly those having slow rises and fast decays (e.g. [7]). In this respect, it has been suggested that the precession of the jet might play an important role in the formation of its time microstructure and this could be valid in both long and short gamma-ray bursts (e.g. $[8,9,10,11])$. In [11] a model for precessing jets has been based on spin-induced precession of a neutrino-cooled massive disk. We will use this model to obtain information about the inner system from the GRB light-curves.

Our goal here is the study of another expected byproduct of disk precession, namely the production of gravitational waves. Strong gravitational radiation is likely in several situations: when the gravitational collapse originating the burst is non-spherical, in the presence of strong inhomogeneities in the accretion disk, or, for short GRBs, as a result of the spiral merging of compact objects (e.g. $[12,13])$.

In the next sections we will present a model for the production of a gamma-ray light curve by a central engine consisting of a massive black hole, a transient accretion disk and a jet. Considering Lense-Thirring precession we will then compute gravitational waves emitted by such a source and finally discuss their possible detection.

\section{Accretion disk and spin-induced precession}

In any model for a transient accretion disk formed after massive collapse or merging of compact objects, it is reasonable to consider it to be initially misaligned with the resulting black hole. As first noticed by Lense and Thirring in the general case [14], in the central engine of a GRB this misalignment will cause disk precession. This phenomenon is originated in the dragging suffered by the inertial frames near a rapidly spinning black hole.

When the Mach number inside the disk is below 5, which seems to be the case in most GRBs, it is likely that the disk precesses approximately like a rigid body, i.e., it presents no warping [15]. Of course, the precession of the disk should lead to the precession of the jets, yielding a likely source of temporal micro-variability in the gamma-ray signal [11]. 
The typical disk accretion rate, ranging from $\sim 0.1$ to $10 M_{\odot} \mathrm{s}^{-1}$, is expected to significantly vary in the outer part of the disk. In contrast, for the inner disk a constant accretion rate should be a valid approximation (e.g. $[16,17])$. The conservation of mass, falling with a velocity $v_{r} \simeq$ $r \sqrt{G M_{\mathrm{bh}} r^{-3}}$ at a distance $r$ from the black hole axis, is given by

$$
\dot{M}=-2 \pi r v_{r} \Sigma(r)
$$

where $\Sigma(r)=2 \rho(r) H(r)$ is the surface density, $H(r)$ is the disk half-thickness, and $\rho(r)$ is the mass density of the disk. Now, conservation of angular momentum and energy can be used to numerically compute $\Sigma(r)$ and $H(r)$, considering that the heat generated by friction can be balanced by advection and neutrino emission [11].

Without nutation, the precession period of the disk $\tau_{\mathrm{p}}$ can be related to its surface density by $[18,11]$

$$
\tau_{p}=\int_{0}^{2 \pi} \frac{L_{\mathrm{d}}}{T_{\mathrm{d}}} \sin \theta d \phi=2 \pi \sin \theta \frac{L_{\mathrm{d}}}{T_{\mathrm{d}}}
$$

where the values of the disk angular momentum $L_{\mathrm{d}}$ and applied precessional torque $T_{\mathrm{d}}$ are

$$
\begin{aligned}
& L_{\mathrm{d}}=2 \pi \int_{R_{\mathrm{ms}}}^{R_{\mathrm{out}}} \Sigma(r) \Omega_{\mathrm{k}}(r) r^{3} d r, \\
& T_{\mathrm{d}}=4 \pi^{2} \sin \theta \int_{R_{\mathrm{ms}}}^{R_{\mathrm{out}}} \Sigma(r) \Omega_{\mathrm{k}}(r) v_{p, \theta}(r) r^{3} d r .
\end{aligned}
$$

The relativistic Keplerian angular velocity reads

$$
\Omega_{\mathrm{k}}(r)=\frac{c^{3}}{G M_{\mathrm{bh}}}\left[\left(\frac{r}{R_{\mathrm{g}}}\right)^{3 / 2}+a_{*}\right]^{-1},
$$

where $a_{*}$ is the spin parameter, $R_{\mathrm{g}}=G M_{\mathrm{bh}} / c^{2}$ the gravitational radius, and

$$
v_{p, \theta}=\frac{\Omega_{k}(R)}{2 \pi}\left[1-\sqrt{1 \mp 4 a_{*}\left(\frac{R_{\mathrm{g}}}{r}\right)^{1 / 2}+3 a_{*}^{2}\left(\frac{R_{\mathrm{g}}}{r}\right)^{2}}\right]
$$

is the nodal frequency resulting from the perturbation of a circular orbit in the Kerr metric [19]. Since the action of viscous torques leads to the alignment of the very inner part of the accretion disk with the black hole equator plane [20], the precessing part of the disk ends at an outer radius $R_{\text {out }}$, extending from an inner radius $R_{\mathrm{ms}}=\xi_{\mathrm{ms}} R_{\mathrm{g}}$, where

$$
\xi_{\mathrm{ms}}=3+A_{2} \mp\left[\left(3-A_{1}\right)\left(3+A_{1}+2 A_{2}\right)\right]^{1 / 2},
$$

with

$$
A_{1}=1+\left(1-a_{*}^{2}\right)^{1 / 3}\left[\left(1+a_{*}\right)^{1 / 3}+\left(1-a_{*}\right)^{1 / 3}\right]
$$

and

$$
A_{2}=\left(3 a_{*}^{2}+A_{1}^{2}\right)^{1 / 2}
$$

The minus sign in $\xi_{\mathrm{ms}}$ corresponds to prograde motion $\left(a_{*}>0\right)$, whereas the plus sign corresponds to retrograde motion $\left(a_{*}<0\right)$. 


\section{Gravitational waves from Lense-Thirring precession}

The gamma-ray luminosity produced by a relativistic jet depends on the observation angle $\psi$ as given by [9]

$$
L(\psi)=\frac{27}{4}\left[e^{-0.6 \Psi(x)}-\frac{8}{9}\right]\left[e^{-0.3 \Psi(x)}-e^{-6.3 \Psi(x)}\right] \frac{d \Psi(x)}{d x} .
$$

Here, $\psi$ is the angle between the jet and the observer, $x=10 \sin \psi$ and $\Psi(x)=\frac{1}{6} \ln \left(1+4 x^{2}\right)$. The intrinsic time dependence of the light signal, on the other hand, might be characterized by a FRED (Fast Rise and Exponential Decay) function,

$$
I(t)=N_{I}\left(1-e^{-\frac{t}{\tau_{\text {rise }}}}\right)\left\{\frac{\pi}{2}-\tan ^{-1}\left[\frac{t-\tau_{\text {plat }}}{\tau_{\text {dec }}}\right]\right\},
$$

where $N_{I}$ is a normalization constant such that the maximum of the signal corresponds to unity. The initial rise, plateau, and decay timescales are $\tau_{\text {rise }}, \tau_{\text {plat }}$, and $\tau_{\text {dec }}$ respectively.

In order to make our predictions we shall consider two specific GRBs, the short GRB 990720 and the long GRB 990712. For these events, we use $F(t)=I(t) \times L(\psi(t))$ to reproduce the observed light curves. Here, the angle between the jet and the observer is time-dependent because the azimuthal angle of the jet is $\phi(t)=2 \pi\left(t / \tau_{\text {prec }}\right.$ ) as a result of the disk/jet precession (see Fig.1). Both angles are related by

$$
\cos \psi=\hat{r}_{\text {jet }} \cdot \hat{r}_{\mathrm{obs}}=\cos \phi \sin \alpha \sin \theta_{\mathrm{obs}}+\cos \alpha \cos \theta_{\mathrm{obs}}
$$

Taking this into account, we can obtain the relevant timescales and precession period by the procedure above described. In both cases, the observer is located at $\theta_{\mathrm{obs}}=2^{\circ}$ with respect to the $z$-axis perpendicular to the black hole equator, and we set $\phi_{\mathrm{obs}}$ at $\phi=0$ (see Fig.1).

\subsection{Emission of gravitational waves}

Axis-symmetric bodies (i.e. with inertial moments $I_{1}=I_{2}$ ) in precession emit gravitational waves with an amplitude given by [21, 22]

$$
h_{\mathrm{prec}}(t)=h_{+}(t)+h_{\times}(t),
$$

where

$$
h_{+}(t)=F_{+, 1} \cos \Omega t+F_{+, 2} \cos 2 \Omega t, \quad h_{\times}(t)=F_{\times, 1} \sin \Omega t+F_{\times, 2} \sin 2 \Omega t,
$$

with

$$
\begin{aligned}
& F_{+, 1}=h_{0}^{\prime} \sin 2 \alpha \sin \imath \cos \imath, \quad F_{+, 2}=2 h_{0}^{\prime} \sin ^{2} \alpha\left(1+\cos ^{2} \imath\right) \\
& F_{\times, 1}=h_{0}^{\prime} \sin 2 \alpha \sin \imath, \quad F_{\times, 2}=4 h_{0}^{\prime} \sin ^{2} \alpha \cos \imath,
\end{aligned}
$$

and $h_{0}^{\prime}=-\frac{G}{c^{4}}\left(I_{3}-I_{1}\right) \Omega^{2} / d$. Here, $\alpha$ is the angle between the angular momentum of the disk and that of the black hole (see Fig.1), $\imath$ is the angle between the $z$-axis of the detector and the signal direction of arrival (line of sight), and $d$ is the distance to the radiating body. The principal moments 


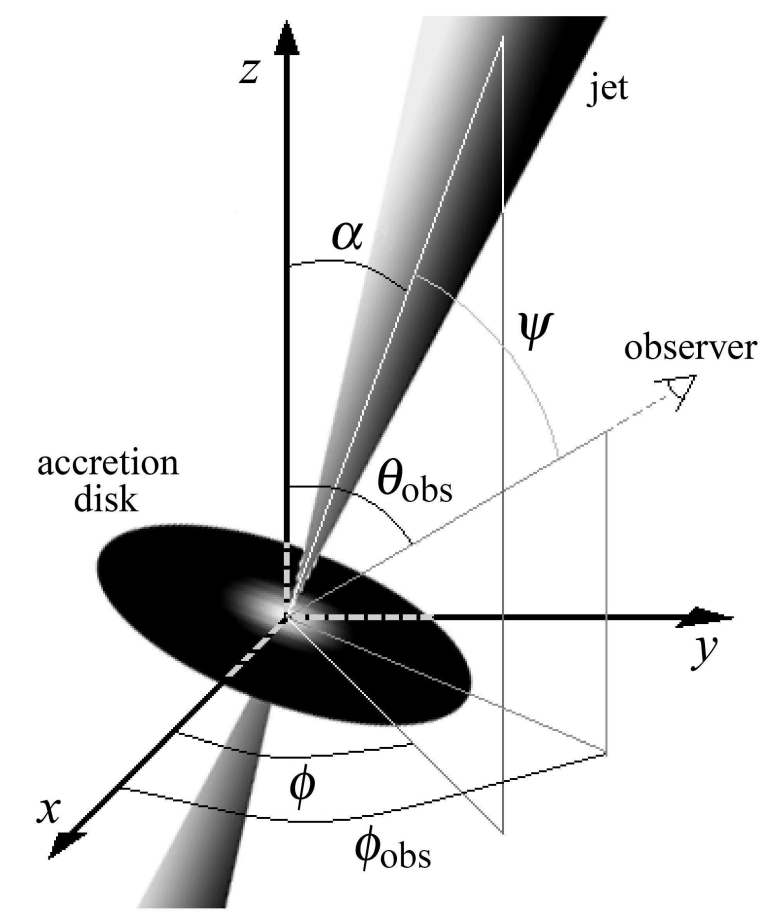

Figure 1: Pictorial scheme of a GRB engine.

of inertia are $I_{3}=\int_{V}\left(x^{2}+y^{2}\right) \rho(r), I_{1}=\int_{V}\left(z^{2}+y^{2}\right) \rho(r)$. The frequency of the gravitational waves $(\mathrm{GW})$ are $f_{1}=\Omega /(2 \pi)$ and $f_{2}=2 \Omega /(2 \pi)$, which are related to the angular momentum of the body by $\Omega=L / I_{1}$.

Since we are dealing with bursting events, the GW signal is expected to be significant for a brief time $\tau_{\text {plat }}$. We therefore modulate the signal given by Eq. (3.4) with a gaussian

$$
h(t)=h_{\text {prec }}(t) e^{-\frac{t^{2}}{2 \tau_{\text {plat }}^{2}}}
$$

as usually done to describe GW signals from bursting sources (e.g. [22, 23]). The angular frequencies that contribute to the waveform of Eq. (3.6) can be better obtained looking at the Fourier transform of Eq.(3.4), $\tilde{h}(\omega)=\tilde{h}_{+}(\omega)+\tilde{h}_{\times}(\omega)$, where

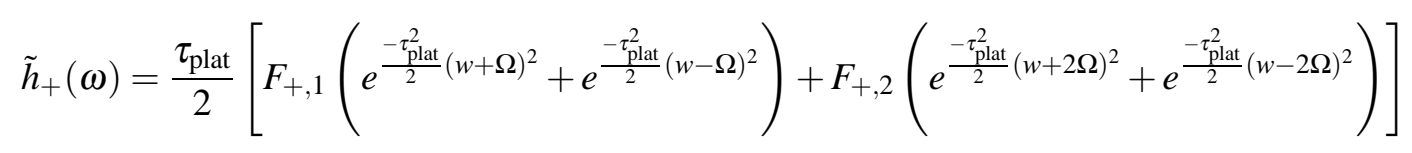

and

$$
\tilde{h}_{\times}(\omega)=\frac{i \tau_{\text {plat }}}{2}\left[F_{\times, 1}\left(e^{\frac{-\tau_{\text {plat }}^{2}}{2}(\omega+\Omega)^{2}}+e^{\frac{-\tau_{\text {plat }}^{2}}{2}(\omega-\Omega)^{2}}\right)+F_{\times, 2}\left(e^{\frac{-\tau_{\text {plat }}^{2}}{2}(\omega+2 \Omega)^{2}}+e^{\frac{-\tau_{\text {plat }}^{2}}{2}(\omega-2 \Omega)^{2}}\right)\right] .
$$

From these expressions it is apparent that the main contributions to the signals are close around $\Omega$ and $2 \Omega$, and the width of the interval is $\tau_{\text {plat }}^{-1}$. Given the typical durations of GRBs, the frequency spread is narrow for all burst except for those with durations much shorter than $1 \mathrm{~s}$. 

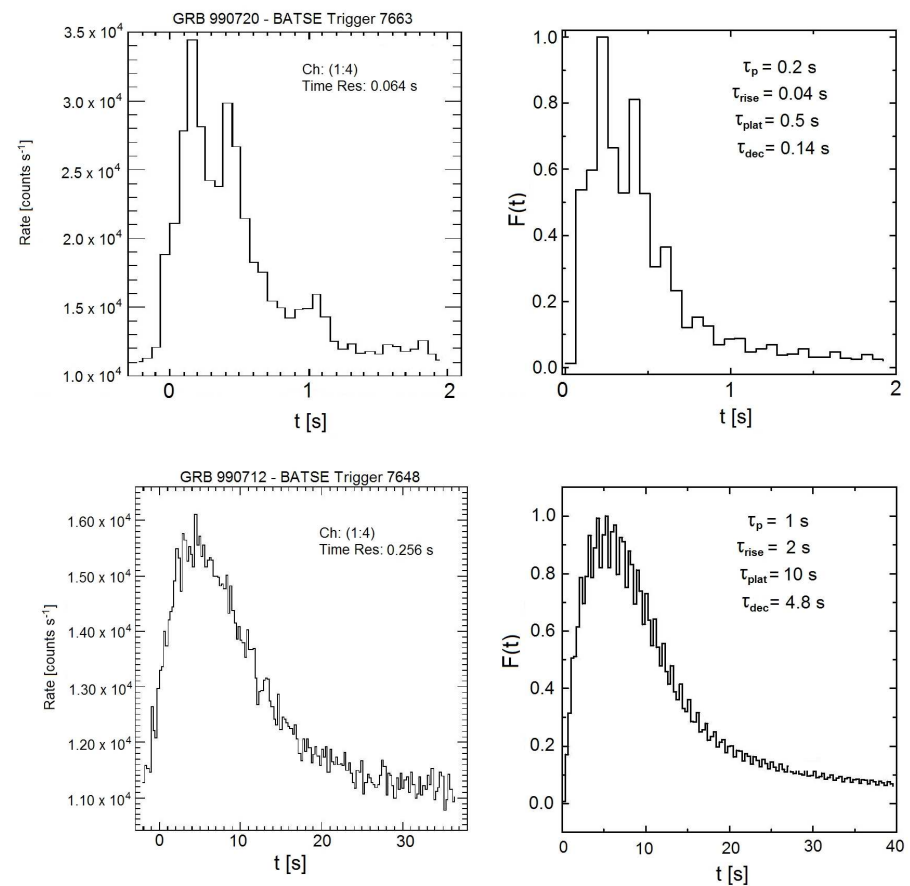

Figure 2: (up) GRB 990720 light curve, experimental (left panel) and model obtained (right panel). (down) GRB 990712 light curve, experimental (left panel) and model obtained (right panel).

\subsection{Numerical predictions of Lense-Thirring gravitational waves}

In order to judge detectability, we compute the root-sum-square (rss) amplitude (e.g. [22, 23]) using Eqs. (3.5)

$$
h_{\mathrm{rss}}(f)=\sqrt{\int_{-\infty}^{\infty} d t\left(h_{+}^{2}+h_{\times}^{2}\right) e^{-\frac{t^{2}}{\tau_{\text {plat }}^{2}}}} .
$$

For illustration, we choose $\imath=45^{\circ}$ and $\alpha=10^{\circ}, 20^{\circ}$. In Fig. 3 we plot the root-sum-square amplitude $h_{\text {rss }}$, as a function of one of the resonant frequencies, $f=\Omega /(2 \pi)$, and also as a function of the outer radius of the inner precessing disk. In the figure we include the expected sensitivity for Advanced LIGO [24]. The parameters used for finding $\Sigma(r)$ and $H(r)$ are $M_{\mathrm{bh}}=3 M_{\odot}, a_{*}=0.1$, a viscosity parameter $\alpha=0.1$, and the different mass loss rates are $\dot{M}=\left\{0.1 M_{\odot} \mathrm{s}^{-1}, 1 M_{\odot} \mathrm{s}^{-1}, 10 M_{\odot} \mathrm{s}^{-1}\right\}$. The distance to the GRB is taken as $d=100 \mathrm{Mpc}$.

Fig. 3 makes clear that there are better chances of detection for accretion rates higher than $1 M_{\odot} \mathrm{s}^{-1}$ and outer radii between $10^{7}$ and $10^{8} \mathrm{~cm}$. Note that when the accretion rate is very high the disk may become advection dominated rather than cooled by neutrino emission [25] but this should not affect the dynamics in the gravitational field. On the other hand, large accretion rates can be only supported in long GRBs, so we conclude that there is only a good prospect for detection of gravitational waves from precessing disks of nearby $(d<100 \mathrm{Mpc})$ long events. Such events are likely related to the death of very massive stars, so the host galaxies should have active star forming regions.

Two low-luminosity long GRBs (980425 and 060218) were already observed at distances of $\sim 40 \mathrm{Mpc}$ and $\sim 130 \mathrm{Mpc}$, respectively [26]. The local rate of long GRBs is estimated to be $\sim 200$ 

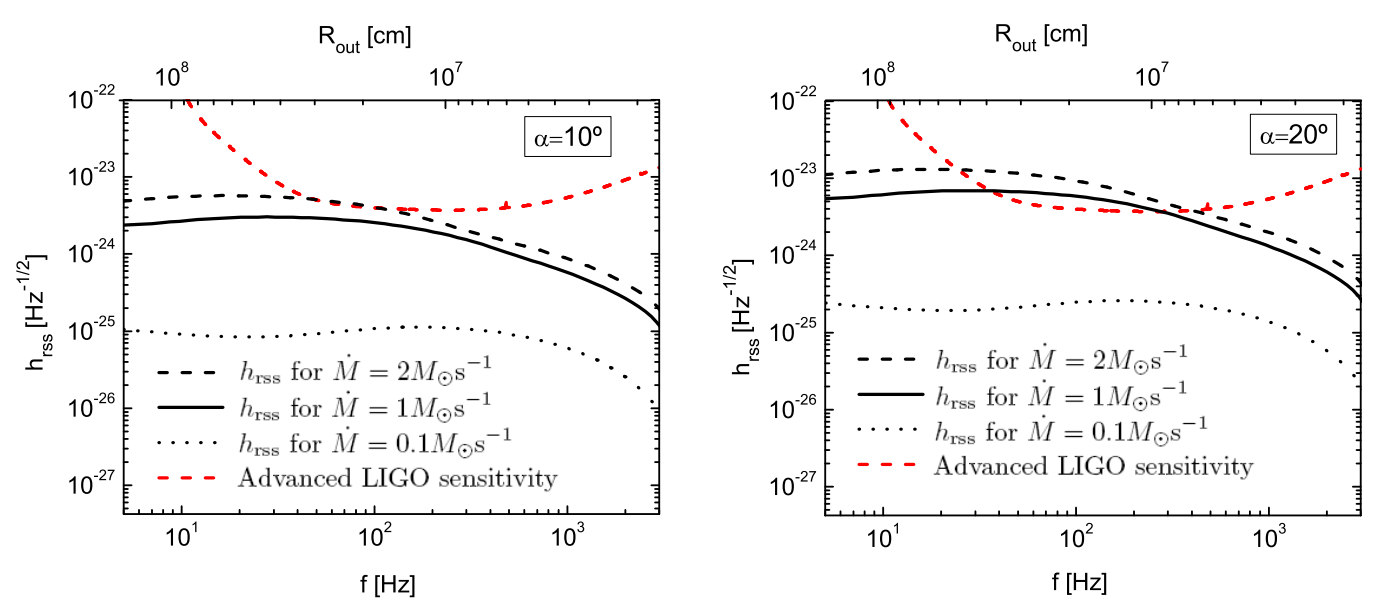

Figure 3: Gravitational wave $r s s$ amplitude for different accretion rates and Advanced LIGO sensitivity (red dashed line) as a function of the gravitational wave frequency, for $\alpha=10^{\circ}$ (left panel) and $\alpha=20^{\circ}$ (right panel). The corresponding outer radius of the accretion disk is indicated in the upper horizontal axis.

$\mathrm{Gpc}^{-3} \mathrm{yr}^{-1}$ (e.g. [27, 28]) and INTEGRAL has detected a significant ratio of faint GRBs which are inferred to be local [29]. As a consequence, this work shows that in the near future the detection of precessing disks in the central engines of GRBs should be possible through their gravitational emission [30]. The detection of one event of this class can be used to test the Lense-Thirring effect in the strong field limit.

Acknowledgements: O.A. Sampayo is acknowledged for useful comments. This research was supported by the Argentine Agencies CONICET and ANPCyT through grants PIP 0078 and PICT2007-00848 BID 1728/OC-AR. GER acknowledges additional support from the Spanish MICINN under grant AYA2010-21782-C03-01.

\section{References}

[1] S. E. Woosley, (1993) ApJ 405, 273.

[2] R. Mochkovitch, M. Hernanz, J. Isern \& X. Martin, (1993) Nature 361, 236.

[3] S. Kobayashi, T. Piran \& R. Sari, (1997) ApJ 490, 92.

[4] F. Daigne, \& R. Mochkovitch, (1998) MNRAS 296, 275.

[5] D. Guetta, M. Spada \& E. Waxman (2001) ApJ 557, 399.

[6] S. Heinz, \& M. C. Begelman, (1999) ApJ 527, L35.

[7] G. E. Romero, D. F. Torres, I. Andruchow \& L. A. Anchordoqui, (1999) MNRAS 308, 799.

[8] E. G. Blackman, I. Yi \& G. B. Field, (1996) ApJ 473, L79.

[9] S. F. Portegies-Zwart, C. H. Lee \& H. K. Lee, (1999) ApJ 520, 666.

[10] D. Fargion, (1999) in: D. Kieda, M. Salamon \& B. Dingus (eds.), 26th ICRC, OG2.3.14, p.32

[11] M. M. Reynoso, G. E. Romero \& O. A. Sampayo, (2006) A\&A 454, 11. 
[12] S. Mineshinge, T. Hosohawa, M. Machida \& R. Matsumoto, (2002) PASJ 54, 655.

[13] S. Kobayashi \& P. Mészáros, (2003) ApJ 589, 861.

[14] J. Lense \& H. Thirring, (1918) Phys. Z. 19, 156.

[15] R. P. Nelson \& J. C. B. Papaloizou, (2000) MNRAS 315, 570.

[16] R. Popham, S. E. Woosley \& C. Fryer, (1999) ApJ 518, 356.

[17] T. Di Matteo, R. Perna \& R. Narayan, (2002) ApJ 579, 706.

[18] S. Liu, \& F. Melia, (2002) ApJ 573, L23.

[19] S. Kato, (1990) PASJ 42, 99.

[20] J. M. Bardeen \& J. A. Petterson, (1975) ApJ 195, L65.

[21] M. Zimmermann \& E. Szedenits, (1979) Phys. Rev. D 20, 351.

[22] M. Maggiore, (2008) Gravitational waves. Volume 1: Theory and Experiments, Oxford University Press, New York.

[23] F. Acernese, et al., (2008) Class. Quantum Grav. 25, 225001.

[24] D. Shoemaker, (LIGO Scientific Collaboration), 2010, LIGO document T0900288-v3, https://dcc.ligo.org/cgi-bin/DocDB/ ShowDocument?docid=2974

[25] T. Liu, W.-M.Gu, L. Xue, S.-S. Weng \& J.-F. Lu, (2008) ApJ 676, 545.

[26] A. Corsi \& P. Mészáros, (2009) ApJ 702, 1171.

[27] E. Liang, B. Zhang, F. Virgili \& Z. G. Dai, (2007) ApJ 662, 1111.

[28] F. J. Virgili, E.-W. Liang \& B. Zhang, (2009) MNRAS 392, 91.

[29] S. Foley, S. McGlynn, L. Hanlon, S. McBreen \& B. McBreen, (2008) A\&A 484, 143.

[30] G. E. Romero, M. M. Reynoso \& H. R. Christiansen, (2010) A\&A 524, A4. 